\title{
A New Health Condition Detection Method for Planetary Gears Based on Modified Distributed Compressed Sensing and Multiscale Symbol Dynamic Entropy
}

\author{
Zhe Wu $\oplus^{1},{ }^{1}$ Qiang Zhang $\oplus^{2},{ }^{2}$ Zeyu Ma, ${ }^{1}$ Jialong Lu, ${ }^{1}$ and Zhiying Qin ${ }^{1}$ \\ ${ }^{1}$ School of Mechanical Engineering, Hebei University of Science and Technology, Shijiazhuang 050018, China \\ ${ }^{2}$ Key Laboratory of Vehicle Transmission, China North Vehicle Research Institute, Beijing 100072, China \\ Correspondence should be addressed to Qiang Zhang; qiangzh36@gmail.com
}

Received 26 November 2020; Revised 22 January 2021; Accepted 18 February 2021; Published 30 March 2021

Academic Editor: Dr Mahdi Mohammadpour

Copyright (C) 2021 Zhe Wu et al. This is an open access article distributed under the Creative Commons Attribution License, which permits unrestricted use, distribution, and reproduction in any medium, provided the original work is properly cited.

Planetary gear transmission system is an important transmission part of large machinery and is prone to failure. Aiming at the problem of how to extract fault information from vibration signals of nonlinear and nonstationary planetary gearboxes, a performance degradation evaluation index of planetary gearboxes based on improved distributed compressed sensing and modified multiscale symbolic dynamic entropy (DCSMDE) is proposed. DCSMDE combines distributed compression sensing with modified multiscale symbol dynamic entropy and solves the problem of strong nonlinearity and strong vibration signal coupling of the planetary transmission system from the homologous signals of multiple sensors. A distributed compression sensing parameter optimization algorithm based on Rényi entropy is proposed, which uses improved distributed compression sensing technology to simultaneously sample, compress, and denoise the multisource vibration data of rotating machinery. DCSMDE is used to calculate the reconstructed signal, extract the features with higher recognition characteristics, and use the change trend of the DCSMDE value to judge the working status of the planetary gearbox. Experimental results show that DCSMDE can be applied to dynamic evolution and fault identification of mechanical systems and accurately classify actual fault signals. It provides a new idea for the classification of planetary gear faults and the recognition of performance degradation.

\section{Introduction}

Planetary gear transmission systems have many advantages such as high transmission ratios and high transmission efficiency. They are widely used in wind power equipment, military equipment, heavy-duty vehicles, and large mechanical equipment. In recent years, the wind industry has developed rapidly, and wind power generation has become a very important resource for all countries. With the rapid growth of wind turbine assembly capacity, many problems have been exposed behind the vigorous development of the wind power industry. There are frequent cases of major safety accidents and casualties caused by the failure of the planetary gear transmission system, especially caused by the failure of the unit equipment. Increasing failure rate has led to a rapid increase in health management and operation and maintenance costs $[1,2]$. Therefore, the safety and stability of the planetary gear transmission system are very important. Using predictive maintenance can greatly reduce the regular maintenance inspections that lead to nonvalue-added maintenance functions [3]. The reliability research and health management of wind turbines are highly valued by scientific researchers in the field of wind power in various countries. It is very necessary to monitor wind turbines around the clock [4].

The sun gear and planetary gears are the most fragile components in planetary gearboxes [5]. In various gearboxes, the probability of gear failure is more than $50 \%$. Most gear failures are caused by partial damage to the gears, such as spalling, cracks, wear, and broken teeth on the tooth surface. Therefore, the dynamic assessment of gear local damage degradation has important practical significance for 
the stable and safe operation of planetary gear trains [6]. The vibration signal can directly reflect the operating information of the planetary gear system. However, when the planetary gear system fails, it will cause the complex nonlinear internal excitation of the system [7]. The nonlinear vibration of the planetary gear system coupled with its own internal and external excitation such as transmission vibration and meshing vibration makes the vibration signal of the planetary gear system contain complex, nonlinear, and nonstationary components [7-9]. These characteristics lead to a low success rate of condition monitoring and fault diagnosis of planetary gear systems. Planetary gearbox has a complex dynamic response due to its special gear transmission structure, which increases the difficulty of extracting the fault feature of the planetary gearbox [8]. Traditional methods use time domain and frequency domain characteristic indexes to study and judge the running state [7]. These methods cannot deeply understand the gear dynamic system that generates vibration signals, and they have poor sensitivity in identifying early gear defects $[9,10]$.

The time-frequency analysis method can simultaneously reflect the frequency components and time-varying characteristics of the signal. Researchers at home and abroad use wavelet transform, empirical mode decomposition, and local mean decomposition and other classical nonlinear signal processing methods to monitor the fault state of planetary gear trains. Many new methods have been proposed for identification and recognition, such as ensemble empirical mode decomposition and energy separation [11], adaptive stochastic resonance [12], improved wavelet transform algorithm [13, 14], time synchronization average [15], continuous vibration separation [16], and variational modal decomposition $[17,18]$.

In order to enhance the signal transmission efficiency and solve the problems of acquisition signal distortion and noise doping, Donoho [19] proposed compressed sensing in 2004, which optimized the reconstructed signal after sparse representation of the unidirectional channel signal. More information is extracted from less data, but the reconstruction process of this algorithm cannot refer to the correlation between homologous signals, and the signal reconstruction has a poor ability to retain characteristic information compared to the original signal. In 2005, Duarte et al. [20] proposed the concept of joint sparse signal based on the relevance of the joint sparsity of the signal set and established the distributed compressed sensing theory (distributed compressed sensing). Compared with singlesignal compressed sensing, distributed compressed sensing can use each sensor to independently measure the internal and intercorrelation of the signal to jointly reconstruct the signal, increase the accuracy of the reconstructed signal, reduce the influence of noise signals, and the homologous signals are used to reconstruct while reducing the amount of data required for reconstruction and reducing computational pressure $[21,22]$.

Distributed compressed sensing is widely used in various fields because of its accurate reconstruction characteristics. Zhou et al. [23] applied distributed compressed sensing technology to the underwater acoustic multiband system to improve the tap signal channel. Reference [24] proposed an energy-efficient underwater acoustic sensor data aggregation network based on distributed compressed sensing. Reference [25] proposed a joint detection and tracking algorithm for multistatic radar systems based on distributed compressed sensing to improve radar detection performance. $\mathrm{Bi}$ et al. proposed a distributed compressed sensing tomographic synthetic aperture radar imaging method based on matrix compensation. In [26], distributed compressed sensing is also applied in the field of communication information $[27,28]$ and image reconstruction $[29,30]$. At the same time, many kinds of optimization algorithms and new strategies have been proposed in recent years [31-34]. In this paper, distributed compressed sensing is used to improve the accuracy of fault analysis and increase the signal-to-noise ratio in the process of signal reconstruction.

How to effectively find the weak signs of degradation from the vibration signals of the faulty parts of the planetary gear train and accurately identify its working status is very important. Entropy is one of the most powerful tools to detect the uncertainty, regularity, and dynamic characteristics of time series. Shannon entropy, sample entropy, transfer entropy, and permutation entropy can measure the complexity of nonlinear system time series [35, 36]. Chen et al. [37] proposed a rolling bearing fault diagnosis method based on wavelet packet energy entropy, which improves the real-time performance of fault detection. Reference [38] used the sensitivity of amplitude-aware displacement entropy (AAPE) to signal amplitude and frequency to achieve coarse-grained time series fault feature extraction. Qin et al. [39] proposed a new method of planetary gear transmission system fault diagnosis based on M-band flexible wavelet transform and spectral negative entropy. The K-SVD algorithm proposed by Qin recovers repeated transient signals through an optimized dictionary. This method can extract fault features faster than the traditional K-SVD method [40].

Compared with the existing methods such as SE and PE [41], SDE (Symbol Dynamic Entropy) has obvious advantages in sensitivity to amplitude change signals and the ability to resist noise. References [42-44] proposed on the basis of SDE improved multiscale symbol dynamic entropy (MMSDE). The MMSDE method discards the coarsegrained process and uses the moving average process instead, which avoids the introduction of uncertain entropy when analyzing short-term time series [45]. In feature extraction, features with higher discrimination can be extracted. MMSDE has obvious advantages in detecting dynamic changes and high computational efficiency. Scholars at home and abroad have conducted in-depth studies on the evaluation of the failure state of the planetary gear transmission system.

Li et al. [46] proposed an improved Vold-Kalman filter combined with multiscale sample entropy to monitor the condition of planetary gearboxes under nonstationary conditions and verified the effectiveness of the method through experimental signals. Reference [42] proposed a planetary gearbox fault diagnosis method based on MMSDE. In order to extract fault features at different multiscales, this method combines the moving average process with symbolic 
dynamic entropy. The experimental results show that the method can successfully identify the local damage of the sun gear, planetary gear, and ring gear. Li et al. [47] combined Variational Mode Decomposition (VMD) and Generalized Composite Multiscale Symbolic Dynamic Entropy (GCMSDE) and used GCMSDE to extract fault information from the IMF generated by VMD. This method can distinguish 7 types of partial failures of the sun gear, planetary gear, and ring gear of the planetary gearbox.

The above research provides an important basis for the state monitoring and identification of planetary gear trains, but there are still many problems to be solved urgently. Most of the above methods extract state characteristic values from a single time series collected by a single sensor, cannot analyze the coupling and correlation between signals, and cannot comprehensively evaluate and evaluate the true operating state of the planetary transmission system.

This paper proposes a new evaluation index DCSMDE for the condition monitoring and fault diagnosis of the planetary transmission system. DCSMDE combines distributed compressed sensing with improved multiscale symbol dynamic entropy and uses improved distributed compressed sensing technology to synchronously sample, compress, and reduce noise from multisource vibration data of rotating machinery and then perform MMSDE calculations on the denoised signal. DCSMDE is used to extract features with higher distinguishing characteristics from the vibration acceleration signals of rotating machinery in different directions. Judge the operating status of the planetary gearbox under real-time detection information. The main contributions of this paper are as follows:

(1) Synchronous noise reduction of multiple sensors: use distributed compressed sensing to denoise and reconstruct the signal, analyze the spatial correlation, sparseness, and joint sparse characteristics of the multidimensional vibration signal of the multistage planetary gear system, and analyze the distributed compressed sensing technology. Compared with the data before processing, the multisource vibration data of rotating machinery reduces noise interference. The signal is accurately reconstructed with fewer measuring points and measurement times, which improves the retention of fault information. Through nonlinear joint reconstruction, the processed signal is sparse and robust at the same time, avoiding the redundancy of collected information. It can realize synchronous sampling, compression, and noise reduction of multiple data at each sensor node.

(2) A parameter optimization algorithm for distributed compressed sensing is proposed. For analyzing the effect of distributed compressed sensing decoupling noise signals, the observation matrix is established as a curve of the Rényi entropy parameter, and the macromechanical characteristics and the microdigital signal are established through the Rényi entropy curve to test the influence of the observation matrix of different parameter dimensions on the noise reduction effect.
(3) MMSDE has obvious advantages in detecting dynamic changes and high computational efficiency. Introduce MMSDE into the field of planetary gearbox fault diagnosis, and use MMSDE to deal with the identification of planetary gear fault types. The MMSDE method discards the coarse-grained process and uses the moving average process instead, which avoids the introduction of uncertain entropy when analyzing short-term time series. And in fault detection, it can extract the features with high distinguishability. Based on the advantage that multiscale entropy can extract fault features at different multiscales, this method can more comprehensively extract signal fault features, which provides a technical reference for new fault diagnosis methods.

(4) This paper combines distributed compressed sensing with improved multiscale symbol dynamic entropy. An evaluation index for the degradation characteristics of planetary gearboxes based on distributed compressed sensing and improved multiscale symbol dynamic entropy is proposed. Compared with existing methods, the proposed method starts with multisensor homologous signals, trying to solve the strong nonlinearity and strong coupling characteristics of the vibration signal of the planetary transmission system. It not only considers the autocorrelation of the signal but also fully considers the homologous signal. This is an important innovation in this paper.

The chapters of this paper are arranged as follows: In Section 2, a planetary gear transmission degradation characteristic evaluation index DCSMDE is proposed, and the basic principles and detailed steps of the method are introduced. In Section 3, the fault data of planetary gears are used to verify the proposed method. In Section 3.2, the observation matrix parameter optimization algorithm of distributed compressed sensing is proposed, and the data analysis of the original signal and the method of using Rényi entropy to determine the best parameters of distributed compressed sensing are given. In Section 3.3, the application of the proposed method in the planetary gear transmission system is studied and discussed in detail using experimental signals, and the effectiveness of the method is verified. The conclusions are presented in Section 4.

\section{Theory}

2.1. Distributed Compressed Sensing. Distributed compressed sensing theory plays a good role in fault type diagnosis and running condition monitoring of rotating machinery. The unprocessed original signal cannot directly reflect the fault information, and in the process of obtaining the vibration signal, the fault information is easy to be masked by excessive noise. To reduce the signal noise interference and achieve the purpose of real-time fault information monitoring of rotating machinery to be tested, the distributed compressed sensing method is used to obtain the reconstructed signal with a higher signal-to-noise ratio and 
improve the accuracy of fault degradation feature evaluation. The flow of the distributed compressed sensing algorithm is as follows.

The $j$-path signals collected by the sensor are used as the original signal, the Fourier orthogonal sparse basis $\Psi$ is selected, and the sparse vector $s$ is used to represent the sparse coefficient of the original signal $X$ after sparse representation $[48,49]$.

$$
y_{j}=\Phi_{j} x_{j}=\Phi_{j} \Psi_{j} s_{j}=A_{j} s_{j}
$$

On the joint sparse model JSM-2, there are $j$ original signals with common sparsity in the same Fourier orthogonal sparse basis [50]. By using the correlation characteristics of multichannel signals, the measurement matrix $S_{M * J}$ whose dimension is far less than the original signal length is obtained by joint observation of all signal sources. The same observation matrix is used for sparse observation of these signals. $z_{j}$ represents the unique sparse part of each signal, and the sparsity under the same sparse basis is $K_{j}$.

$$
\begin{aligned}
x_{j} & =\Psi s_{j}, \quad j \in\{1,2,3, \ldots, J\}, \\
\left\|\theta_{j}\right\|_{0} & =K_{j} .
\end{aligned}
$$

Fourier transform the signal $X$ of dimension $M$ and the coefficient after the signal transformation is recorded as $S$ $(n)$. The $K$ coefficients with the largest amplitude are selected for retention, and the remaining coefficients can be regarded as 0 . In the multichannel signal joint reconstruction, each column and the observation matrix calculate the inner products separately and then sum these inner products.

$$
\begin{aligned}
r_{j} & =y_{j}, \\
\widehat{I} & =\varnothing, \\
A_{0} & =\varnothing, \\
t & =1 .
\end{aligned}
$$

The maximum value of the sum of the absolute values of the inner product of multiple signals is taken as the coordinate of the most relevant column index in this iteration. The coefficient is added to the joint support set $\widehat{I}$.

$$
\begin{aligned}
& \lambda_{t}=\arg \max _{t} \sum_{j=1}^{J}\left|A_{t}^{T} r_{J}\right| ; \\
& \widehat{I}_{t}=\widehat{I}_{t-1} \cup\left\{\lambda_{t}\right\}, \\
& A_{t}=A_{t-1} \cup \alpha_{\lambda_{t}} .
\end{aligned}
$$

For each signal, the sparse coefficient $\theta_{i * J}$ is obtained by finding an approximate solution on the column for each signal.

The residual value of each column is updated according to each iteration until $t \leq K$. When the residual values meet the preset conditions, the iteration is terminated to obtain $K$ residual values, and the minimum binomial solution of $y_{j}=$ $A_{t} s_{j t}$ is calculated.

$$
\begin{aligned}
& \widehat{s}_{j t}=\arg \min _{s_{j}}\left\|y_{j}-A_{t} s_{j t}\right\|=\left(A_{t}^{T} A_{T}\right)^{-1} A_{t}^{T} y_{j}, \\
& r_{j t}=y_{j}-A_{t} \widehat{s}_{j t}=y_{j}-A_{t}\left(A_{t}^{T} A_{T}\right)^{-1} A_{t}^{T} y_{j} .
\end{aligned}
$$

After getting the predicted sparse vector coefficient $\widehat{s}_{j}$, the sparse matrix can be used to reconstruct the signal, and the reconstructed signal which is sensed by distributed compressed sensing under the simulated fault state of broken teeth can be obtained.

Distributed compressed sensing technology can effectively reduce noise and extract signal features, which is the premise of establishing reliable fault state classification and recognition. The processed distributed compressed sensing time series signal can effectively increase the accuracy of monitoring. We find that there is a certain regularity between the Rényi entropy and the scale parameters, and with the transformation of the parameter scale, the variation trend chart of Rényi entropy can reflect different parameter states to a certain extent. To obtain a better-compressed sensing effect and meet the requirements of signal denoising in the following steps, Rényi entropy is used to explore the dimension of the observation matrix suitable for the process of mechanical vibration signal denoising.

Rényi entropy can accurately reflect the nonlinear characteristics and coupling relationship of system states. In this paper, a parameter optimization algorithm for distributed compressed sensing is proposed, and the Rényi entropy is introduced into the parameter optimization of distributed compressed sensing [51]. The Rényi entropy versus scale curve is used to evaluate the retention degree of different observation matrix dimensions for the reconstructed signal state feature information. Under the dimension of the optimal observation matrix, the original signal filters and reduces the interference noise error through the distributed compressed sensing step, which lays a good foundation for the follow-up analysis [52].

The influence of different dimension parameters of observation matrix on the noise reduction effect of distributed compressed sensing is calculated, and the optimal observation matrix parameters are obtained, so that the signal reconstructed by distributed compressive sensing can contain more feature information and avoid the damage of fault feature information by distributed compressive sensing, so as to achieve better noise reduction effect [52-54].

$$
\left\{S_{\tau}(\rho)=\frac{1}{1-\tau} \log \operatorname{tr}\left(\rho^{\tau}\right), \quad \tau \in Z_{+}, \tau \neq 1 .\right.
$$

The distributed compressed sensing of multisource signals in the same state under different observation matrix parameter dimensions is carried out, and the state density matrix based on the original data and the reconstructed signal is established. Then, the multiscale Rényi entropy calculation is carried out to study the degree of fault feature retention of the reconstructed signal and the original signal. The parameter optimization algorithm of distributed compressed sensing based on Rényi entropy can measure the distributed compressed sensing effect of multisource vibration data. The method is simple and can accurately obtain 
the state recognition results of distributed compressed sensing effect.

2.2. Modified Multiscale Symbol Dynamic Entropy. The MMSDE method uses a moving average process instead of a coarse-grained process, which overcomes the problem of low accuracy when analyzing short time series by MSDE. At the same time, multiscale entropy can extract tomographic features on different scales. Starting from multisensor homologous signals, it can extract signal state features more comprehensively and has obvious advantages in detecting signal dynamic changes and computing efficiency. The MMSDE algorithm flow is as follows [42].

First, perform a moving average process on the time series $X\{x(k), k=1,2, \ldots, N\}$ to obtain a multiscale time series. Use equation (7) to divide the time series into several moving average time series by the scale factor of $\tau$.

$$
y_{j}^{\tau}=\frac{1}{\tau}\left(\sum_{i=j}^{j+\tau-1}\right) x_{i}, \quad 1 \leq j \leq N-\tau+1,
$$

where $\tau$ represents the scale factor in multiscale analysis.

Then use the SDE algorithm to calculate the SDE value of each moving average time series $\left\{y_{j}^{\tau}\right\}$. The SDE algorithm steps are as follows.

The MEP method is used to complete the symbolization, and the amplitude domain of the time series is divided into $\varepsilon$ units. Each element corresponds to a unique cell. The symbolic time series $Z\{z(k), k=1,2, \ldots, N\}$ can be obtained.

The embedding vector is constructed according to the symbol time series, and the potential state mode probability is calculated. Get a series of embedding vectors:

$$
Z_{i}^{m, \lambda}\{z(j), z(j+\lambda), \ldots, z(j+(m-1) \lambda)\}, \quad j=1,2, \ldots, N-(m-1) \lambda .
$$

The probability of the potential state pattern $P\left(q_{a}^{\varepsilon, m, \lambda}\right)$ is as follows [43]:

$$
P\left(q_{a}^{\varepsilon, m, \lambda}\right)=\frac{\left\{j: j \leq N-(m-1) \lambda, \operatorname{type}\left(Z_{j}^{\varepsilon, m, \lambda}\right)=q_{a}^{\varepsilon, m, \lambda}\right\}}{N-(m-1) \lambda},
$$

where type $\left(Z_{j}^{\varepsilon, m, \lambda}\right)$ represents the mapping from symbol space to state mode space. $\|\{j: j \leq N-(m-1) \lambda$, "type" $\left.\left(Z_{j}^{\varepsilon, m, \lambda}\right)=q_{a}^{\varepsilon, m, \lambda}\right\} \|$ represents the cardinality of the set.
Construct state transition and calculate the probability of state transition. When the state pattern $q_{a}^{\varepsilon, m, \lambda}\left(a=1,2, \ldots, \varepsilon^{m}\right)$ is observed, the state transition probability is the conditional probability of the symbol $\sigma_{b}(b=1,2, \ldots, \varepsilon)$. And the conditional probability satisfies the condition $\sum_{b=1}^{\varepsilon} P\left(\sigma_{b} \mid q_{a}^{\varepsilon, m, \lambda}\right)=1$.

SDE is defined as the sum of state entropy and state transition entropy, as follows [42]:

$$
\operatorname{SDE}(X, m, \lambda, \varepsilon)=-\sum_{a=1}^{\varepsilon^{m}} P\left(q_{a}^{\varepsilon, m, \lambda}\right) \cdot \ln P\left(q_{a}^{\varepsilon, m, \lambda}\right)-\sum_{a=1}^{\varepsilon^{m}} \sum_{b=1}^{\varepsilon} P\left(q_{a}^{\varepsilon, m, \lambda}\right) \cdot \ln P\left(q_{a}^{\varepsilon, m, \lambda}\right) \cdot P\left(\sigma_{b} \mid q_{a}^{\varepsilon, m, \lambda}\right)
$$

Among them, $m$ is the embedding dimension, $\lambda$ is the time delay, and is the number of symbols $\varepsilon$. The maximum value of $\operatorname{SDE}(X, m, \lambda, \varepsilon)$ is $\ln \left(\varepsilon^{m+1}\right)$. Then, the normalized SDE of $\ln \left(\varepsilon^{m+1}\right)$ can be expressed as

$$
\operatorname{SDE}(X, m, \lambda, \varepsilon)=\frac{\operatorname{SDE}(X, m, \lambda, \varepsilon)}{\ln \left(\varepsilon^{m+1}\right)},
$$

$0 \leq \operatorname{SDE}(\varepsilon, m) \leq 1$, and the larger the $\operatorname{SDE}$ value, the more random and irregular the time series. The smaller the SDE value, the more regular and periodic the time series. And the MMSDE can be expressed as $\operatorname{MMSDE}(X, \tau, m, \lambda, \varepsilon)=\operatorname{SDE}$ $\left(y_{j}^{\tau}, m, \lambda, \varepsilon\right)$.

In this paper, a new evaluation index based on improved distributed compressed sensing multiscale symbol dynamic entropy (DCSMDE) is proposed. The main process is as follows:

(1) A three-way vibration acceleration sensor is arranged at the key position of the planetary gearbox to synchronously collect the vibration acceleration signals in $x, y$, and $z$ directions.

(2) Distributed compressed sensing of three-dimensional vibration acceleration signals is carried out, and sparse decomposition is carried out by using the joint sparsity between homologous signals. The optimal solution equation is used to obtain the sparse coefficient, and the reconstructed signal is obtained through the sensing matrix.

(3) In order to study the coupling effect of distributed compressed sensing with different scales of measurement matrix, an optimization algorithm of measurement matrix parameters is proposed. The Rényi entropy curves with parameter scale $\tau$ transformation under different observation matrix dimensions are established to judge the degree of feature retention of reconstructed signals under different observation matrix dimensions. 
(4) Through the moving average process, the signal is transformed into multiscale time series to extract the signal features more comprehensively. MEP method is used to complete the symbolization. The amplitude domain of time series is divided intocunits. According to the symbolic time series, the embedded vector is constructed and the potential state mode probability is calculated. MMSDE is the sum of state entropy and state transition entropy. MMSDE value of the reconstructed signal is calculated and fault analysis results are observed. The flow of DCSMDE is shown in Figure 1.

\section{Experimental Research}

3.1. Experiment Introduction. Using the multistage planetary gear transmission system fault diagnosis test bench, five types of faults including gear broken teeth, tooth surface wear, gear tooth cracks, tooth surface pitting, and missing teeth were simulated [42]. The experimental platform is shown in Figure 2. The entire experimental system includes a control system, servo motor, multistage planetary gearbox, and magnetic powder brake $[55,56]$. A number of three-way acceleration sensors are installed on the planetary gearbox to synchronously collect various operating states of the planetary gearbox, and the sampling frequency is set to $12800 \mathrm{~Hz}$ $[57,58]$.

Figure 3 shows the time domain and frequency spectrum of the vibration signal of the planetary gearbox in the health state.

3.2. Data Preprocessing. The purpose of this paper is to explore whether the distributed compressed sensing and the improved multiscale symbolic dynamic entropy model are suitable for the expected fault types. We simulate the operating conditions of rotating machinery under normal and four fault conditions and obtain three directional vibration acceleration signals to judge the connection of the relevant Rényi entropy map to the microfactors and the overall fault condition. The joint sparse signal set model is constructed by the signals of three different directions in the same position of the rotating machinery (as shown in Figure 4).

The Fourier transform basis is selected as the sparse basis to ensure that the signal satisfies the sparse condition. Using the Gaussian random matrix as the observation matrix, the high-dimensional signal is projected into the low-dimensional vector, and the observed signal matrix as shown in Figure 5 is obtained as the input condition of the synchronous orthogonal matching and tracking algorithm.

Taking the vibration signal of the broken tooth fault state as an example, we carry on the Fourier transform of the collected discrete signal with $j$ length, and the coefficient after the signal transform is recorded as $S(n)$, which selects the largest coefficient among the $K$ amplitudes to retain. The determinant coefficients of the rest of the transfer matrix can be regarded as 0 . At this time, the support set of the three groups of signals is obtained and processed by merging. The joint orthogonal matching pursuit algorithm can be used for information reconstruction, and the reconstructed sparse signal $\widehat{s}_{j}$ is shown in Figure 6 . The reconstructed signal after distributed compression sensing in the simulated fault state of broken teeth is obtained by sparse matrix, and the result is shown in Figure 7.

Through the above steps, we can get the reconstructed signal set of distributed compressed sensing in fault state and normal state. The distributed compressed sensing method is used to reconstruct the vibration signals in three directions, and the autocorrelation and cross-correlation between the signals are used. It can effectively achieve the effect of synchronous noise reduction and multisource noise reduction.

The influence of different observation matrix dimension parameters on the noise reduction effect of distributed compressed sensing is calculated, and the optimal observation matrix parameters are obtained, so that the signal reconstructed by distributed compressed sensing can accommodate more characteristic information, so as to achieve a better noise reduction effect. The Rayleigh entropy method is used to distinguish the reconstructed signals of different observation dimension parameters in the macrostate. Because the same signal is processed by different observation matrix dimensions, the results are usually different. In order to ensure the better information characteristics of the obtained information, the Rayleigh entropy changes of different observation matrix dimensions are observed and the optimal observation matrix dimension is selected, so as to increase the amount of characteristic information carried by the reconstructed signal and improve the computational efficiency. Different observation matrix dimensions are selected to calculate the Rayleigh entropy of the original data, and the results are shown in Figure 8.

As shown in Figure 8, it can be seen that when $M \in[10$, 250], the change trend of Rényi entropy is the same, when $\tau \in[0,0.4]$, the entropy shows a downward trend, when the interval $\tau \in[0.4,0.9]$, the entropy value rapidly increases to the maximum, and when $\tau \in[0.9,1.1]$, the entropy decreases sharply and reaches a negative interval, after which the curve gradually tends to be stable and converges to a negative value. The different parameters are only different at the two peaks $\tau=[0.4,1.1]$. The entropy change trend of the observation matrix dimension $M \in 250$ is inverted relative to the previous interval, and the curve is the opposite trend, indicating that the signal characteristics have an impact on the original signal of the macrostate when the observation matrix dimension is reconstructed when $M \in[200,250]$.

With reference to the two parameters, when the Rényi entropy time delay scale $\tau \in[0,5]$, the entropy of each state will increase and maintain a certain order, representing the information correlation characteristics under the Rényi entropy time delay scale. On the other hand, the curve corresponding to the dimension $M$ of different observation matrix changes as a whole; until the dimension of the observation matrix changes to a certain value, the change trend of entropy is reversed.

The smaller the amplitude of Rényi entropy is, the more the reconstructed signal can reflect the original signal feature vector. According to the principle of distributed compressed 


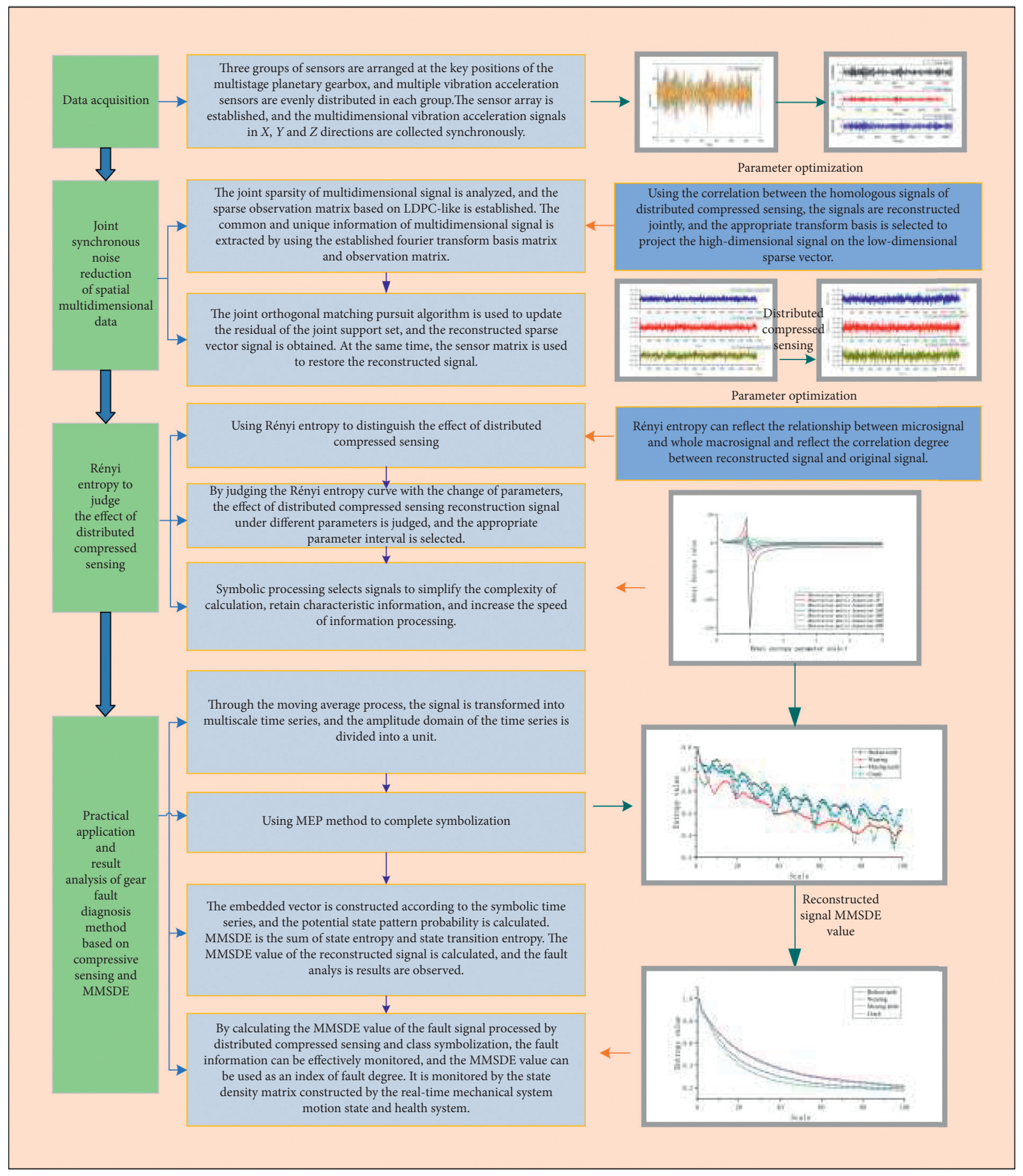

Figure 1: Process of fault analysis method based on distributed compressed sensing multiscale permutation entropy (PE).

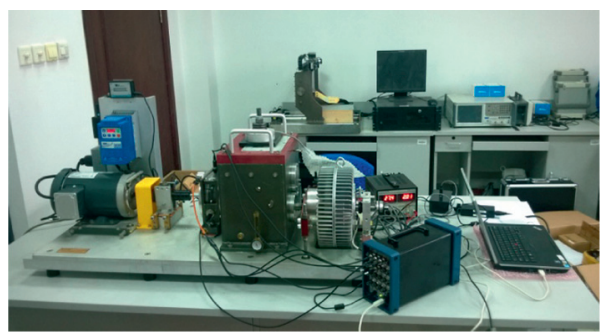

Figure 2: Fault diagnosis test bench for planetary gear box. 


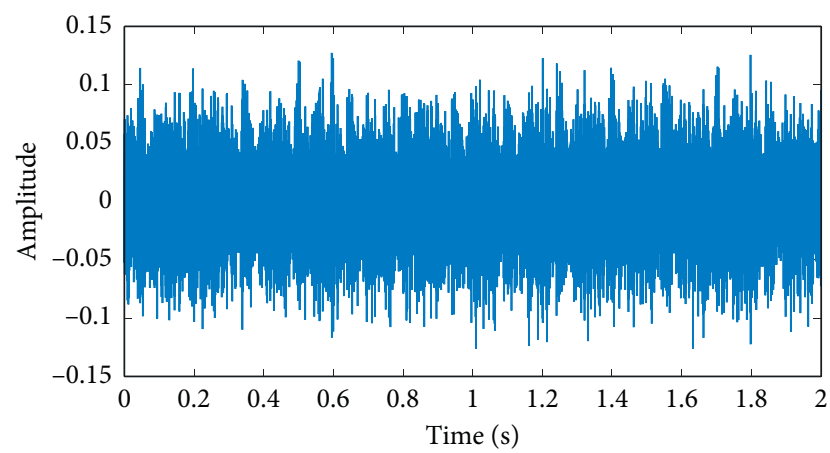

(a)

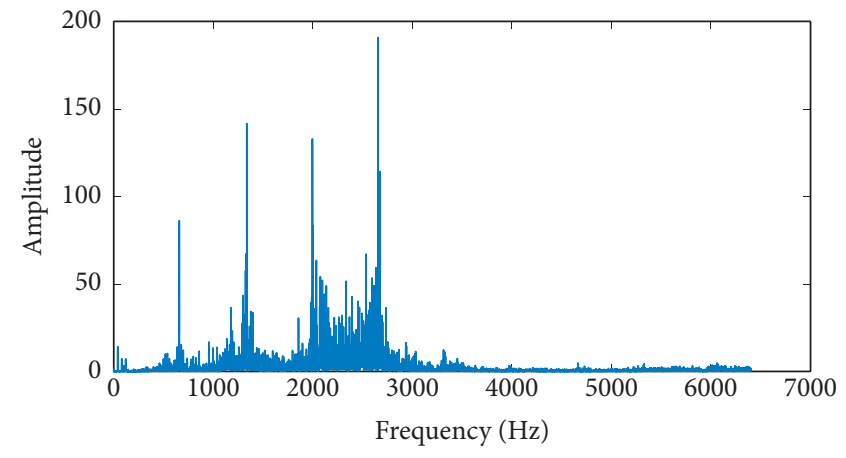

(b)

FIgURE 3: Time domain waveform and spectral diagram of vibration signal of planetary gearbox in healthy state. (a) Time domain waveform. (b) Spectral diagram.
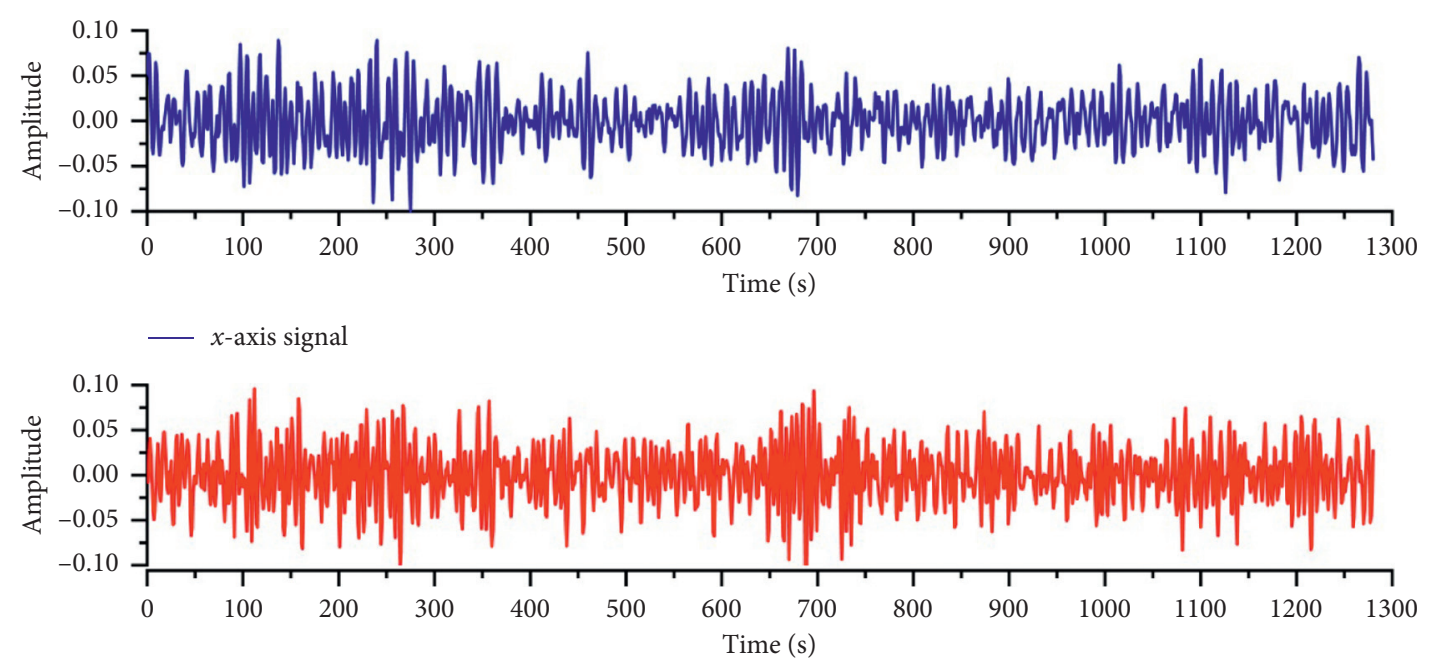

$y$-axis signal

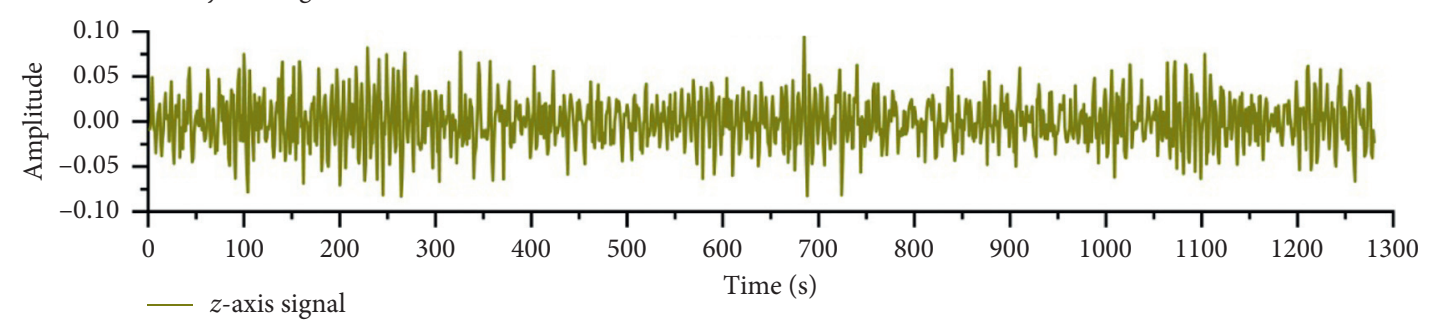

FIgURE 4: Amplitude curve of the original signal in three axial directions.

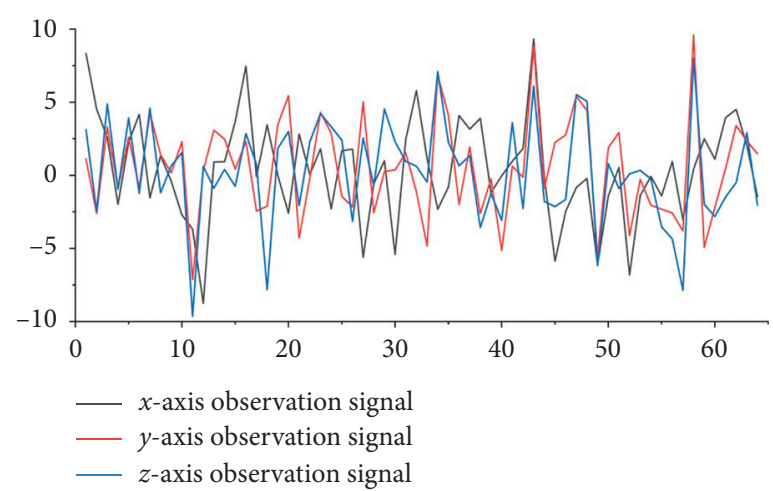

Figure 5: Observation signal matrix after sparse transformation. 

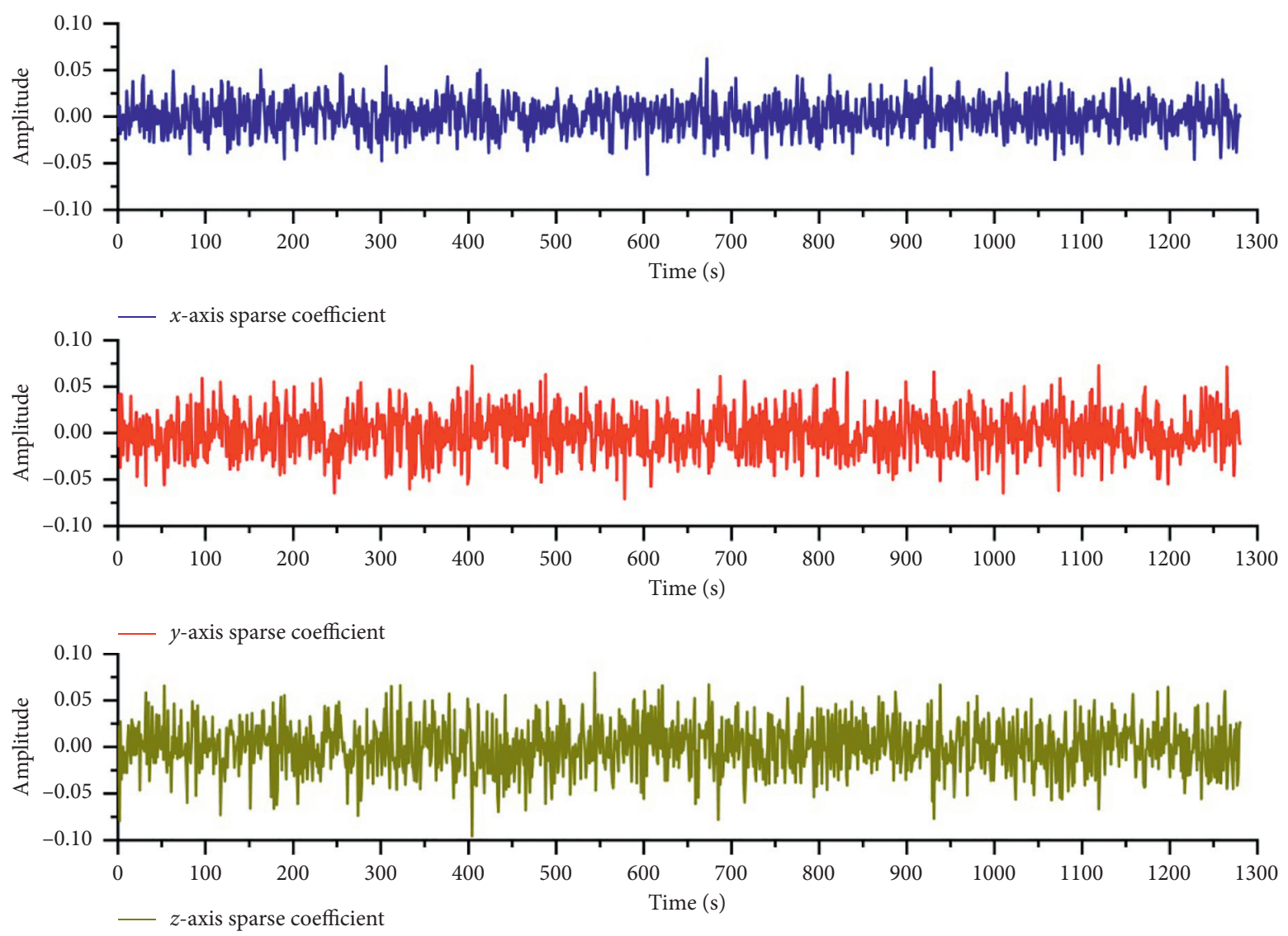

Figure 6: Sparse vectors in three axial directions.

sensing, the observation matrix dimension should be much smaller than the signal dimension, and the observation effect is the best when the observation dimension reaches the interval of $M \in[200,250]$. When $M \in[0,200]$, the peak value of the Rényi entropy image increases rapidly with the change of parameters, which means that the distortion of the reconstructed signal increases with the decrease of scale, so as to determine the suitable parameters and lay a good foundation for follow-up calculation.

3.3. Fault Data Analysis of Planetary Gearbox Based on $D C S M D E$. The planetary gearbox is a complex mechanical system. When the planetary gearbox is in working condition, the signal obtained by the sensor installed on the box has the characteristics of high complexity of spectrum characteristics and low signal-to-noise ratio, especially in the early stage of failure. The energy of the fault signal is very small and it is easy to be submerged in the background noise. The preprocessing process of the vibration signal requires an effective reduction in the complexity of the signal and the loss of characteristic information, so that the subsequent processing and calculation efficiency are improved. Therefore, the key to fault diagnosis and status recognition is to extract the characteristic information of the fault from the vibration signal.
First, perform MMSDE analysis on the original multisource signals of the planetary gearbox under multiple operating states. The results are shown in Figure 9. As shown in the figure, the curve distinctions corresponding to various fault states are not obvious. The classification effect is poor, and there are multiple intersections between the fault state curves. The five types of curves show a downward trend as a whole, and the fault curves are bent, with large changes, and all show periodic and multipeak phenomena. The distinguishing effect of various fault states basically does not change with the scale. Among them, the periodicity of the crack fault state is the most obvious, and the wear fault curve is relatively flat.

Table 1 shows the maximum, minimum, and effective values of various fault MMSDE values. As shown in Table 1, the largest peak drop of the four fault states is broken tooth, which is 0.4142 . The effective value corresponding to broken teeth is the largest, which is 0.5826 , while the effective value corresponding to wear is the smallest, which is 0.5162 . The result of MMSDE analysis using the original signal is poor. This shows that the interference signal and noise contained in the nonlinear and nonstationary vibration signal have a great influence on the experimental signal, so it is necessary to adopt a reasonable noise reduction plan for the signal. Taking into account the autocorrelation of the data itself and the cross-correlation between the data, distributed 

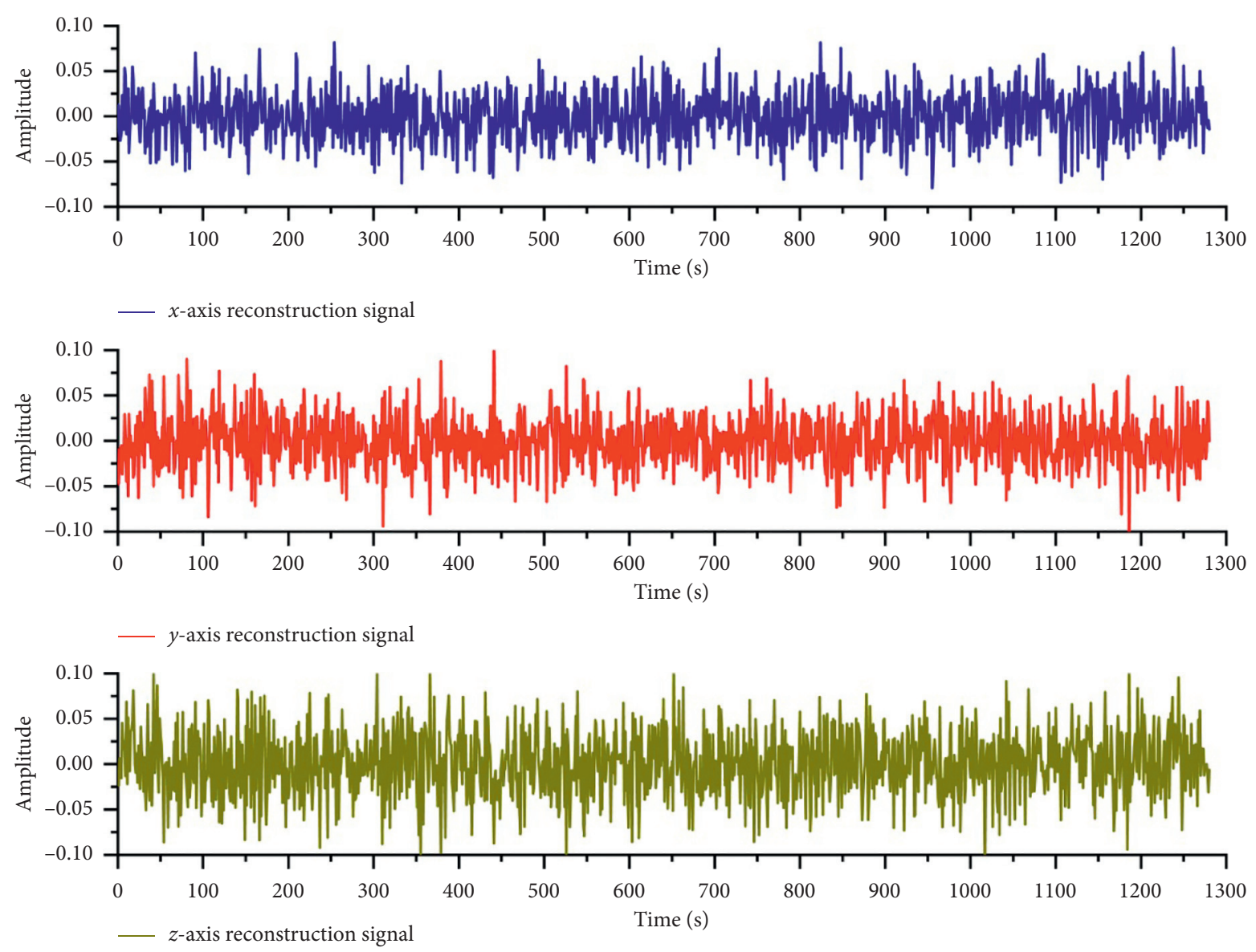

FIgURE 7: Three-axis reconstruction signal.

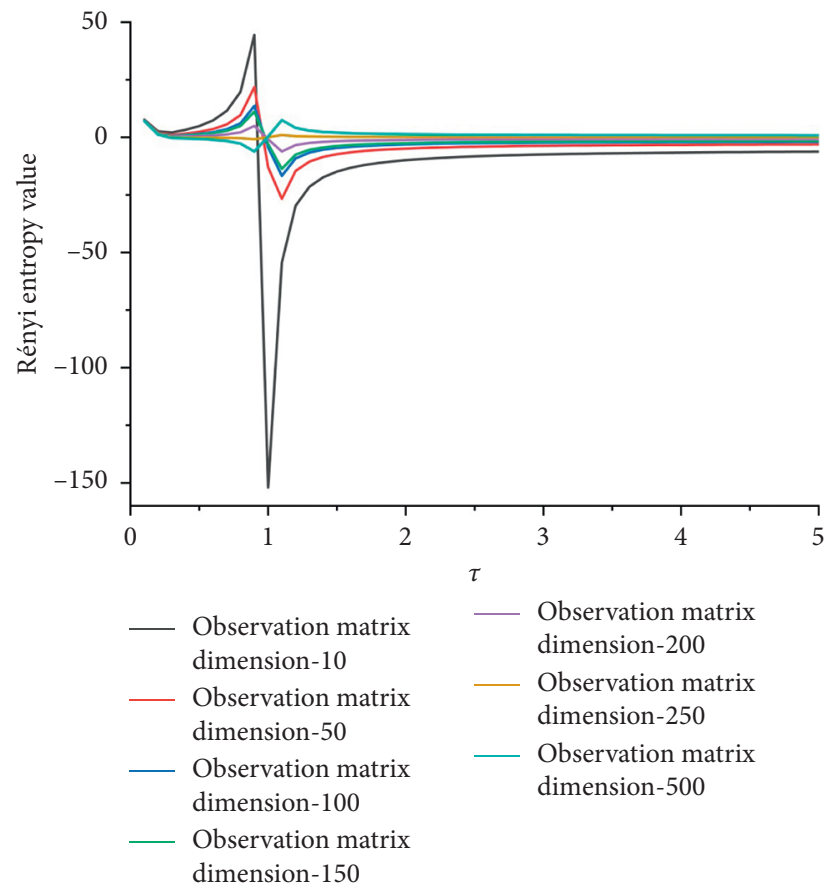

FIgURE 8: Transformation trend of Rényi entropy with parameter scale. 


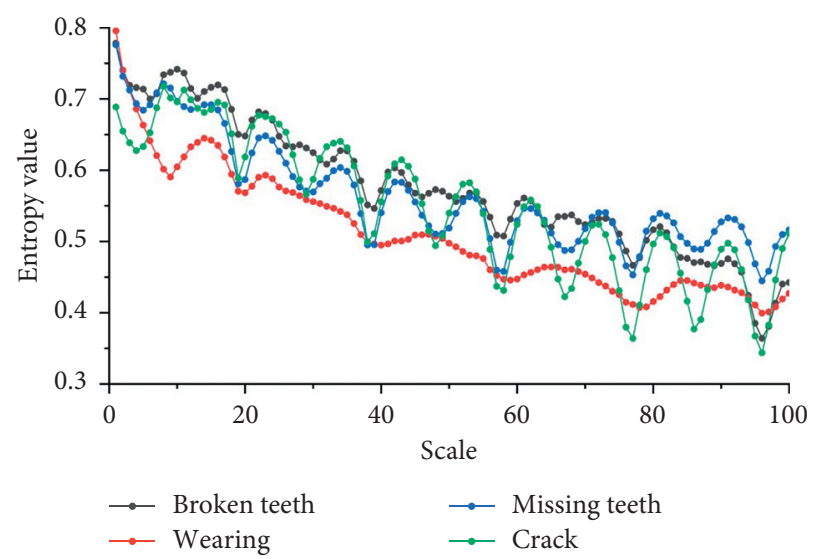

Figure 9: The original signal calculated MMSDE value changes with scale.

compressed sensing is used to process multisource time series ( $x, y$, and $z$ three directions). Take the data after compressed sensing in the $x$ direction to analyze the MMSDE value, and the results are shown in Figure 10.

As shown in Figure 10, the periodicity of the multiscale entropy curve calculated from the signal after the distributed compression sensing process disappears. The entropy curve becomes a smooth downward trend. When the scale factor is small, especially when $\tau \leq 4$, the entropy value of the five states has a larger downward trend. When the scale factor is large, the entropy value of the four states has a slower downward trend. It can be seen from Figure 10 that the overall downward trend of cracks is the largest, and the wear curve is relatively flat. The entropy curves of the four fault states overlap each other, and the difference is not good. It can be seen that the compressed sensing effect of randomly selected parameters is not ideal, and the choice of parameters has a significant impact on the processing results. Take the data after compressed sensing in the $x$ direction to analyze the DCSMDE value, and the results are shown in Figure 11.

As shown in Figure 11, the periodicity of the multiscale entropy curve calculated from the signal after distributed compressed sensing processing disappears. The entropy curve becomes a smooth downward trend and gradually tends to a fixed value. When the scale factor is small, especially when $\tau \leq 20$, the entropy value of the five states changes greatly, and only when the scale factor is large, the entropy values of the four states become relatively stable, and as the scale factor increases, the multiscale entropy curves gradually tend to a certain value. They all tend to a certain value gradually with the increase of the scale factor, which shows that multiscale entropy not only reflects the complexity of the time series itself but also contains information on other scales of the sequence, which is not possible with SDE. It reflects the superiority of multiscale entropy.

It can be seen from Figure 12 that the entropy curves of the four fault states do not overlap, and the distinction is obvious. Calculate the root mean square values of the DCSMDE of the four states after stability, and observe the relationship between their effective values. The results are shown in Table 2. It can be seen from Table 2 that the
TABLE 1: MMSDE value of original signal.

\begin{tabular}{lcccc}
\hline Fault type & Crack & Broken tooth & Missing teeth & Wear \\
\hline Max & 0.7783 & 0.7955 & 0.7761 & 0.7176 \\
Min & 0.3641 & 0.3996 & 0.4449 & 0.3436 \\
Effective value & 0.5826 & 0.5162 & 0.5697 & 0.5579 \\
\hline
\end{tabular}

effective value of DCSMDE of the vibration signal of different fault types of gears is different. The vibration signal of the fault state, according to the entropy value, is wear$>$ missing teeth $>$ broken teeth $>$ cracks. The sample entropy of missing teeth and wear is relatively large, which shows that the vibration signals of the two are more complex and have a higher degree of irregularity. The sample entropy values of normal and broken teeth are relatively small, indicating that the complexity of their vibration signals is low and the sequence self-similarity is high. Multiscale entropy analyses of wear, missing teeth, broken teeth, and crack fault signal DCSMDE values correspond to a curve in the figure. Due to the introduction of scale factors, the entropy values of gear signals of different fault types are different at different scales. The effective value of DCSMDE in the four states is wear $>$ missing teeth $>$ broken teeth $>$ cracks. This shows that DCSMDE can clearly and intuitively distinguish several types of gear failure states.

Finally, under various operating conditions, permutation entropy (PE) analysis is performed on the original multisource signal of the planetary gearbox. The result is shown in Figure 13. When the time delay $t=1$, the entropy value is small, a sudden change occurs at $t=2$, and the entropy value greatly increases. When $t>2$, the entropy value oscillates around a fixed value, and the entropy value changes greatly. The curves corresponding to various fault states overlap in many places. Wear and cracks are almost overlapped, which is difficult to distinguish. Compared with the DCSMDE classification effect, the PE classification effect is poor, and the intersections between the fault state curves appear periodically. Among them, the entropy of missing teeth fluctuates the most, and the peak appears periodically. The discrimination effect of various fault states basically does not change with the scale. It can be seen that DCSMDE has advantages in multiscale analysis. Compared with traditional 


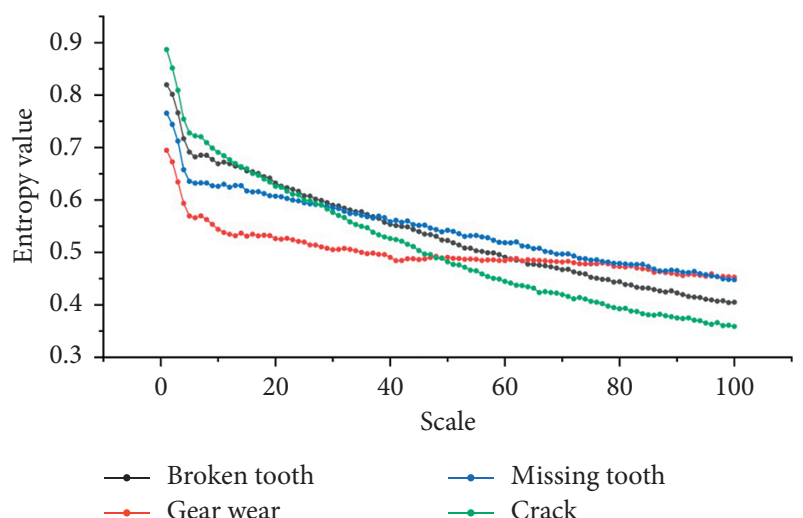

Figure 10: The MMSDE value calculated by the compressed sensing signal changes with the scale.

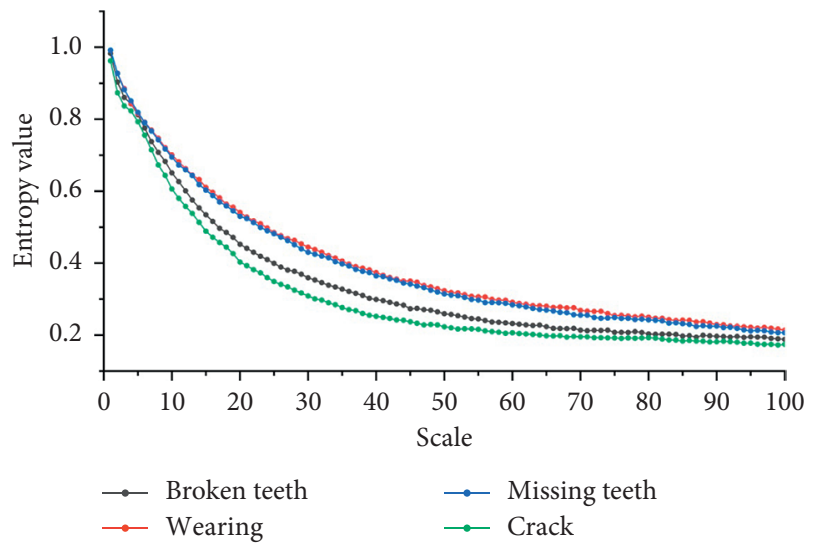

Figure 11: The calculated DCSMDE value of the signal after distributed compressed sensing varies with the scale.

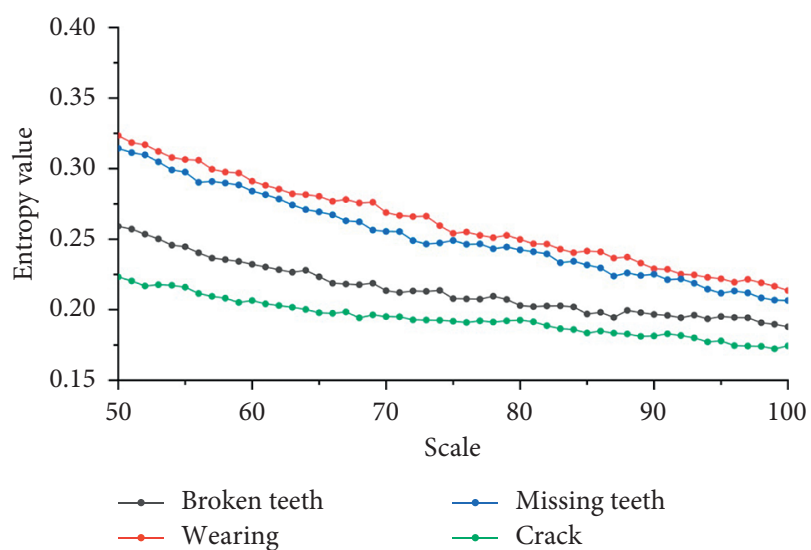

FIgURE 12: DCSMDE value curve after flattening.

TABLE 2: Root mean square values of DCSMDE values for different fault types.

\begin{tabular}{lcccc}
\hline Fault type & Crack & Broken tooth & Missing teeth & Wear \\
\hline Root mean square & 0.2013 & 0.2260 & 0.2692 & 0.2779 \\
\hline
\end{tabular}

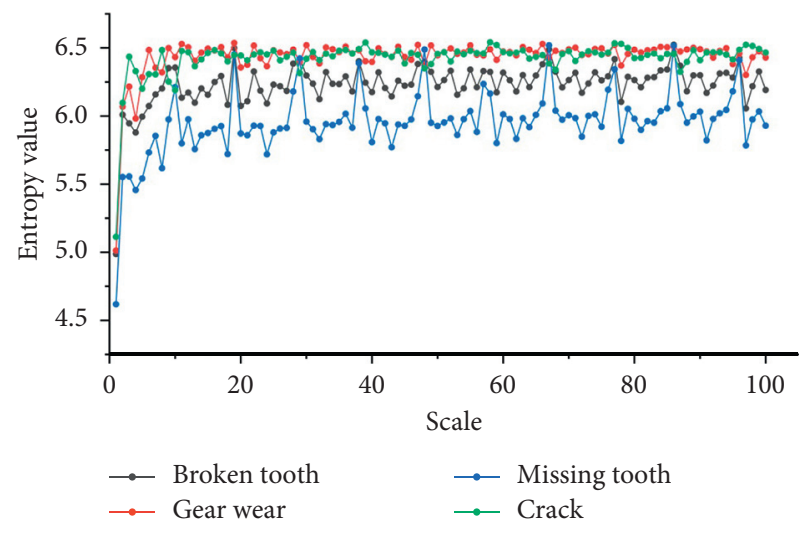

FIGURE 13: The calculated PE value of the signal varies with $t$.

$\mathrm{PE}$, the results are more accurate and the classification effect is better.

\section{Conclusion}

To solve the problem that the vibration signal of planetary gearbox generally has a lot of noise and it is difficult to obtain characteristic information, a new degradation feature evaluation index of planetary gearbox based on DCSMDE is proposed. Through the analysis of the fault signals of missing teeth, broken teeth, wear, and cracks of planetary gearbox, the effectiveness of this degradation index in condition monitoring is verified. The conclusions are as follows:

(1) Combine distributed compressed sensing with MMSDE to form a new evaluation index for planetary gear degradation characteristics. Use the reconstructed signal processed by distributed compressed sensing to calculate the MMSDE value, starting from the multisensor homologous signal, considering the cross-correlation between the homologous signals, and extracting the signal state characteristics more comprehensively. The scale factor is introduced to calculate the effective value of the entropy of different fault states, and the effective value difference of each fault state is about 0.03 . It shows that the method of calculating DCSMDE parameter scale trend can effectively detect the operating conditions of rotating machinery and build the connection between nonlinear mechanical system dynamics and information dynamics. Make it play a role in the modal recognition of nonlinear systems, system dynamics evolution, and fault diagnosis, and accurately classify actual fault signals. Therefore, the planetary gearbox degradation characteristic evaluation index based on DCSMDE is an effective method for planetary gear fault classification.

(2) Distributed compressed sensing can effectively reduce the noise of the signal and increase the proportion of carrying characteristic information, while different observation matrix dimensions will affect the final reconstructed signal. The correlation Rényi 
entropy can well reflect the coupling information between the two nonlinear signals and can reflect the transformation trend of Rényi entropy with the time delay scale. The relationship between the reconstructed signal and the original signal under different observation matrix dimension parameters is derived. Experimental data prove that when the dimension of the observation matrix is $M \in[200,250]$, the effect of the entropy variable function is the best, and the curve is smooth.

(3) Introduce MMSDE into the field of planetary gearbox fault diagnosis, and use MMSDE to deal with the identification of planetary gear fault types. The MMSDE method shortens the length of the time series and overcomes the problem of SDE's low accuracy when analyzing short time series. The MMSDE method is used to process the signal after denoising by distributed compressed sensing, and the moving average process can generate more template vectors. More comprehensive extraction of signal fault characteristics enables MMSDE to provide more accurate entropy estimation. It shows that DCSMDE has obvious superiority in detecting dynamic changes and provides a technical reference for new fault diagnosis methods.

\section{Data Availability}

Some or all data, models, or codes generated or used during the study are available from the corresponding author upon request.

\section{Conflicts of Interest}

The authors declare no conflicts of interest.

\section{Acknowledgments}

This work was partially supported by the National Natural Science Foundation of China, 51705130 and 51706058; S\&T Program of Hebei (20310803D); Natural Science Foundation of Hebei Province (E2020208052); Hebei University Science and Technology Research Youth Fund Project (QN2018168); and Doctor Research Fund of Hebei University of Science and Technology (1181339).

\section{References}

[1] H. Ma and Z. Feng, "Planet bearing fault diagnosis using multipoint optimal minimum entropy deconvolution adjusted," Journal of Sound and Vibration, vol. 449, pp. 235-273, 2019.

[2] T. Wang, Q. Han, F. Chu, and Z. Feng, "Vibration based condition monitoring and fault diagnosis of wind turbine planetary gearbox: a review," Mechanical Systems and Signal Processing, vol. 126, pp. 662-685, 2019.

[3] S. Kumar, D. Goyal, R. K. Dang, S. S. Dhami, and B. S. Pabla, "Condition based maintenance of bearings and gears for fault detection-a review," Materials Today: Proceedings, vol. 5, no. 2, pp. 6128-6137, 2018.
[4] Y. Merizalde, L. Hernández-Callejo, O. Duque-Perez, and R. A. López-Meraz, "fault detection of wind turbine induction generators through current signals and various signal processing techniques," Applied Sciences, vol. 10, no. 21, p. 7389, 2020.

[5] W. Teng, X. Ding, X. Zhang, Y. Liu, and Z. Ma, "Multi-fault detection and failure analysis of wind turbine gearbox using complex wavelet transform," Renewable Energy, vol. 93, pp. 591-598, 2016.

[6] Z. Wu, Q. Zhang, L. Cheng, and S. Tan, "A new method of two-stage planetary gearbox fault detection based on multisensor information fusion," Applied Sciences, vol. 9, no. 24, p. 5443, 2019.

[7] Z. Feng, M. J. Zuo, and F. Chu, "Application of regularization dimension to gear damage assessment," Mechanical Systems and Signal Processing, vol. 24, no. 4, pp. 1081-1098, 2010.

[8] H. Sun, Y. Zi, Z. He, J. Yuan, X. Wang, and L. Chen, "Customized multiwavelets for planetary gearbox fault detection based on vibration sensor signals," Sensors, vol. 13, no. 1, pp. 1183-1209, 2013.

[9] Y. Wang, Z. Fan, H. Liu, and X. Gao, "Planetary gearbox fault diagnosis based on ICEEMD-time-frequency information entropy and VPMCD," Applied Sciences, vol. 10, no. 18, p. 6376, 2020.

[10] W.-T. Du, Q. Zeng, Y.-M. Shao, L.-M. Wang, and X.-X. Ding, "Multi-scale demodulation for fault diagnosis based on a weighted-EMD de-noising technique and time-frequency envelope analysis," Applied Sciences, vol. 10, no. 21, p. 7796, 2020.

[11] Z. Feng, M. Liang, Y. Zhang, and S. Hou, "Fault diagnosis for wind turbine planetary gearboxes via demodulation analysis based on ensemble empirical mode decomposition and energy separation," Renewable Energy, vol. 47, pp. 112-126, 2012.

[12] X.-h. Chen, G. Cheng, X.-l. Shan, X. Hu, Q. Guo, and H.-g. Liu, "Research of weak fault feature information extraction of planetary gear based on ensemble empirical mode decomposition and adaptive stochastic resonance," Measurement, vol. 73, pp. 55-67, 2015.

[13] D. Strömbergsson, P. Marklund, K. Berglund, J. Saari, and A. Thomson, "Mother wavelet selection in the discrete wavelet transform for condition monitoring of wind turbine drivetrain bearings," Wind Energy, vol. 22, no. 11, pp. 1581-1592, 2019.

[14] Y. Kong, T. Wang, and F. Chu, "Meshing frequency modulation assisted empirical wavelet transform for fault diagnosis of wind turbine planetary ring gear," Renewable Energy, vol. 132, pp. 1373-1388, 2019.

[15] J. Yoon, D. He, B. Van Hecke, T. J. Nostrand, J. Zhu, and E. Bechhoefer, "Vibration-based wind turbine planetary gearbox fault diagnosis using spectral averaging," Wind Energy, vol. 19, no. 9, pp. 1733-1747, 2016.

[16] L. Zhang and N. Hu, "Fault diagnosis of sun gear based on continuous vibration separation and minimum entropy deconvolution," Measurement, vol. 141, pp. 332-344, 2019.

[17] V. Sharma and A. Parey, "Extraction of weak fault transients using variational mode decomposition for fault diagnosis of gearbox under varying speed," Engineering Failure Analysis, vol. 107, Article ID 104204, 2020.

[18] Z. Feng, D. Zhang, and M. Zuo, "Planetary gearbox fault diagnosis via joint amplitude and frequency demodulation analysis based on variational mode decomposition," Applied Sciences, vol. 7, no. 8, p. 775, 2017.

[19] D. L. Donoho, "Compressed sensing," IEEE Transactions on Information Theory, vol. 52, no. 4, pp. 1289-1306, 2006. 
[20] M. F. Duarte, S. Sarvotham, D. Baron, M. Wakin, and R. G. Baraniuk, "Distributed compressed sensing of jointly sparse signals," in Proceedings of the 2005 Asilomar Conference on Signals, Systems, and Computers, Pacific Grove, CA, USA, 2005.

[21] B. Muhammad, L. Edmund, and P. Amal, "Compressed sensing-based distributed image compression," Applied Sciences, vol. 2014, no. 4, pp. 128-147, 2014.

[22] L. Li, Y. Fang, L. Liu, H. Peng, J. Kurths, and Y. Yang, "Overview of compressed sensing: sensing model, reconstruction algorithm, and its applications," Applied Sciences, vol. 10 , no. 17 , p. 5909, 2020.

[23] Y. Zhou, A. Song, F. Tong, and R. Kastner, "Distributed compressed sensing based channel estimation for underwater acoustic multiband transmissions," The Journal of the Acoustical Society of America, vol. 143, no. 6, pp. 3985-3996, 2018.

[24] D. Wang, R. Xu, X. Hu, and W. Su, "Energy-efficient distributed compressed sensing data aggregation for clusterbased underwater acoustic sensor networks," International Journal of Distributed Sensor Networks, vol. 12, no. 3, Article ID 8197606, 2016.

[25] J. Liu, F. Lian, and M. Mallick, "Distributed compressed sensing based joint detection and tracking for multistatic radar system," Information Sciences, vol. 369, pp. 100-118, 2016.

[26] H. Bi, B. Zhang, and W. Hong, "Matrix completion-based distributed compressive sensing for polarimetric SAR tomography," Science China Information Sciences, vol. 58, no. 11, pp. 1-3, 2015.

[27] A. Akbarpour-Kasgari and M. Ardebilipour, "Distributed compressed sensing-based channel estimation and pilot allocation for MIMO relay networks," Iranian Journal of Science and Technology, Transactions of Electrical Engineering, vol. 43, no. 1, pp. 159-170, 2019.

[28] S. Patterson, Y. C. Eldar, and I. Keidar, "Distributed compressed sensing for static and time-varying networks," IEEE Transactions on Signal Processing, vol. 62, no. 19, pp. 49314946, 2014.

[29] Y. Wu, Y.-J. Zhu, Q.-Y. Tang et al., “Accelerated MR diffusion tensor imaging using distributed compressed sensing," Magnetic Resonance in Medicine, vol. 71, no. 2, pp. 763-772, 2014.

[30] K. J. Francis, P. Rajalakshmi, and S. S. Channappayya, "Distributed compressed sensing for photo-acoustic imaging," in Proceedings of the 2015 IEEE International Conference on Image Processing (ICIP), IEEE, Quebec City, Canada, 2015.

[31] B. Zhang, Y. L. Liu, and J. X. Zhang, "Distributed compressed sensing based on differential signal sparse model," Application Research of Computers, vol. 10, 2012.

[32] X. Chen, Y. Zhang, and R. Qi, "Block sparse signals recovery algorithm for distributed compressed sensing reconstruction," Journal of Information Processing Systems, vol. 15, no. 2, pp. 410-421, 2019.

[33] H. Liu, G. Hua, and H. Yin, "An intelligent grey wolf optimizer algorithm for distributed compressed sensing," Computational Intelligence and Neuroscience, vol. 2018, Article ID 1723191, 10 pages, 2018.

[34] S. H. Hsieh, W. J. Liang, and C. S. Lu, "Distributed compressive sensing: performance analysis with diverse signal ensembles," IEEE Transactions on Signal Processing, vol. 68, pp. 3500-3514, 2020.
[35] X. Su, H. Liu, and L. Tao, "TF entropy and RFE based diagnosis for centrifugal pumps subject to the limitation of failure samples," Applied Sciences, vol. 10, no. 8, p. 2932, 2020.

[36] D. Sandoval, U. Leturiondo, F. Pozo, and Y. Vidal, "Lowspeed bearing fault diagnosis based on permutation and spectral entropy measures," Applied Sciences, vol. 10, no. 13, p. 4666, 2020.

[37] Y. Chen, T. Zhang, Z. Luo, and K. Sun, "A novel rolling bearing fault diagnosis and severity analysis method," Applied Sciences, vol. 9, no. 11, p. 2356, 2019.

[38] Y. Chen, T. Zhang, W. Zhao, Y. Luo, and H. Lin, "Rotating machinery fault diagnosis based on improved multiscale amplitude-aware permutation entropy and multiclass relevance vector machine," Sensors, vol. 19, no. 20, p. 4542, 2019.

[39] Y. Qin, Y. Mao, B. Tang, Y. Wang, and H. Chen, "M-band flexible wavelet transform and its application to the fault diagnosis of planetary gear transmission systems," Mechanical Systems and Signal Processing, vol. 134, Article ID 106298, 2019.

[40] Y. Qin, J. Zou, and B. Tang, “Transient feature extraction by the improved orthogonal matching pursuit and K-SVD algorithm with adaptive transient dictionary," IEEE Transactions on Industrial Informatics, vol. 16, no. 1, pp. 215-227, 2019.

[41] L. Xiao, Y. Lv, and G. Fu, "fault classification of rotary machinery based on smooth local subspace projection method and permutation entropy," Applied Sciences, vol. 9, no. 10, p. 2102, 2019.

[42] Y. Li, Y. Yang, G. Li, M. Xu, and W. Huang, "A fault diagnosis scheme for planetary gearboxes using modified multi-scale symbolic dynamic entropy and mRMR feature selection," Mechanical Systems and Signal Processing, vol. 91, pp. 295312, 2017.

[43] Y. Li, Y. Yang, X. Wang, B. Liu, and X. Liang, "Early fault diagnosis of rolling bearings based on hierarchical symbol dynamic entropy and binary tree support vector machine," Journal of Sound and Vibration, vol. 428, pp. 72-86, 2018.

[44] Y. Yang, H. Zheng, J. Yin, M. Xu, and Y. Chen, "Refined composite multivariate multiscale symbolic dynamic entropy and its application to fault diagnosis of rotating machine," Measurement, vol. 151, Article ID 107233, 2020.

[45] S.-D. Wu, C.-W. Wu, K.-Y. Lee, and S.-G. Lin, "Modified multiscale entropy for short-term time series analysis," Physica A: Statistical Mechanics and Its Applications, vol. 392, no. 23, pp. 5865-5873, 2013.

[46] Y. Li, K. Feng, X. Liang, and M. J. Zuo, “A fault diagnosis method for planetary gearboxes under non-stationary working conditions using improved Vold-Kalman filter and multi-scale sample entropy," Journal of Sound and Vibration, vol. 439, pp. 271-286, 2019.

[47] Y. Li, G. Li, Y. Wei, B. Liu, and X. Liang, "Health condition identification of planetary gearboxes based on variational mode decomposition and generalized composite multi-scale symbolic dynamic entropy," ISA Transactions, vol. 81, pp. 329-341, 2018.

[48] A. Hormati and M. Vetterli, "Distributed compressed sensing: sparsity models and reconstruction algorithms using annihilating filter," in Proceedings of the 2008 IEEE International Conference on Acoustics, Speech and Signal Processing, IEEE, Las Vegas, NV, USA, 2008.

[49] M. Gharavi-Alkhansari and T. S. Huang, "A fast orthogonal matching pursuit algorithm," in Proceedings of the 1998 IEEE International Conference on Acoustics, Speech and Signal Processing, Seattle, WA, USA, 1998. 
[50] D. Baron, M. F. Duarte, and M. B. Wakin, "Distributed compressive sensing," 2009, https://arxiv.org/abs/0901.3403.

[51] R. L. Frank and E. H. Lieb, "Monotonicity of a relative Rényi entropy," Journal of Mathematical Physics, vol. 54, no. 12, Article ID 122201, 2013.

[52] L. Golshani, E. Pasha, and G. Yari, "Some properties of Rényi entropy and Rényi entropy rate," Information Sciences, vol. 179, no. 14, pp. 2426-2433, 2009.

[53] A. B. Kallin, I. González, and M. B. Hastings, "Valence bond and von Neumann entanglement entropy in Heisenberg ladders," Physical Review Letters, vol. 103, no. 11, Article ID $117203,2009$.

[54] M. B. Hastings, I. González, and A. B. Kallin, "Measuring Renyi entanglement entropy in quantum Monte Carlo simulations," Physical Review Letters, vol. 104, no. 15, Article ID 157201, 2010.

[55] G. Cheng, X. Chen, H. Li, P. Li, and H. Liu, "Study on planetary gear fault diagnosis based on entropy feature fusion of ensemble empirical mode decomposition," Measurement, vol. 91, pp. 140-154, 2016.

[56] Y. Li, G. Cheng, C. Liu, and X. Chen, "Study on planetary gear fault diagnosis based on variational mode decomposition and deep neural networks," Measurement, vol. 130, pp. 94-104, 2018.

[57] M. Kuai, G. Cheng, Y. Pang, and Y. Li, "Research of planetary gear fault diagnosis based on permutation entropy of CEEMDAN and ANFIS," Sensors, vol. 18, no. 3, p. 782, 2018.

[58] Y. Li, G. Cheng, Y. Pang, and M. Kuai, "Planetary gear fault diagnosis via feature image extraction based on multi central frequencies and vibration signal frequency spectrum," Sensors, vol. 18, no. 6, p. 1735, 2018. 